\title{
Évaluer les monnaies a la fin du Moyen-Âge. Une information imparfaite et inégale
}

Marc Bompaire

\section{OpenEdition}

\section{Journals}

Édition électronique

URL : http://journals.openedition.org/ress/210

DOI : $10.4000 /$ ress. 210

ISSN : 1663-4446

Éditeur

Librairie Droz

Édition imprimée

Date de publication : 1 juillet 2007

Pagination : 69-79

ISBN : 978-2-600-01155-6

ISSN : 0048-8046

\section{Référence électronique}

Marc Bompaire, «Évaluer les monnaies a la fin du Moyen-Âge. Une information imparfaite et inégale », Revue européenne des sciences sociales [En ligne], XLV-137 | 2007, mis en ligne le 01 juillet 2010,

consulté le 01 mai 2019. URL : http://journals.openedition.org/ress/210 ; DOI : 10.4000/ress.210 
Marc BOMPAIRE

\section{ÉVALUER LES MONNAIES A LA FIN DU MOYEN-ÂGE Une information imparfaite et inégale}

\section{DIVERSITÉ DES MONNAIES ET DES ÉVALUATIONS}

En 1322, le vicomte de Lomagne versait à Avignon 150000 florins, soit une partie du trésor de son oncle, le pape Clément V, qu'il avait emporté à la mort de celui-ci. La remise de cette somme occupa durant six jours cinq banquiers suivant la Curie, changeurs et agents des compagnies italiennes. Leur travail, attesté par un long procès-verbal, n'avait rien de formel et il se conclut par la réclamation d'un paiement complémentaire puisqu'il y avait un certain nombre de pièces fausses, légères et que l'évaluation (au poids) des quelques lingots ou des monnaies d'or de l'Espagne musulmane et de Castille leur attribuait une valeur sans doute inférieure à celle qu'espérait le vicomte de Lomagne'

Je vous épargne l'inventaire : il s'agit par cet exemple de rappeler ce qu'était le maniement du numéraire. A tous les niveaux, tout paiement est une aventure et le choix du moyen de paiement est sans aucun doute un élément de la négociation du prix. L'évaluation en monnaie de compte, évoquée ci-dessus dans d'autres contributions, avait l'avantage de rendre homogènes et comparables des monnaies diverses et fluctuantes. Il fallait bien de temps à autre en passer par les monnaies réelles, mais à quel taux?

Les comptabilités sont remplies de lignes de pertes (et plus rarement de gains) au «change », c'est-à-dire pour des pièces reçues à un certain cours (en monnaie de compte) et dépensées à un cours inférieur... Il y a donc évaluation et «change» à l'intérieur même du système monétaire français, malgré l'existence d'un cours légal. Les spécialistes qui opèrent à Avignon paraissent s'appuyer sur le contenu de métal précieux, le poids de fin, comme fondement ultime et objectif de leurs évaluations. L'écart entre cours officiel et contenu métallique apparaît à la source des profits des changeurs comme du trésor royal en cette période $\left(\mathrm{XIV}^{\mathrm{e}}\right.$ et $\mathrm{XV}^{\mathrm{e}}$ siècles) où l'administration monétaire ne se privait pas de muer et remuer les monnaies. Il est donc important de comprendre comment l'administration ou les changeurs établissaient leurs évaluations et l'usage qu'en faisaient les simples utilisateurs des pièces.

Comme à Avignon, les changeurs et courtiers présents sur les marchés apparaissent bien souvent comme l'autorité consultée en cas de doute ou de contestation. Je prendrai pour exemple l'aventure, sans doute assez banale, d'un marchand

1 Guérard (1903), p. 53. 
drapier de Barcelone en 1342 qui, passant par Perpignan, y change son numéraire d'argent en anges d'or. Ceux-ci lui sont refusés quelques jours plus tard par un changeur de Toulouse car «ce ne sont pas des anges que fait faire le roi de France» mais des imitations frappées par le roi de Majorque à Perpignan : le changeur propose de les lui reprendre avec une décote sensible. Il est tard, le marchand demande à réfléchir et décampe dans la nuit : il se sait repéré avec des monnaies interdites que les sergents du roi peuvent l'obliger à porter à l'atelier monétaire de Toulouse, à un cours désastreux et avec amende. Dès le lendemain, il achète des draps à Limoux et Fanjeaux (Aude) où il n'y avait apparemment pas de changeurs informés. Au contraire, le courtier de l'opération (qu'il avait dû mettre dans son jeu) abonde dans son sens au moment du paiement et affirme que ces anges sont de bonnes pièces ${ }^{2}$. Les écarts dans les évaluations sont clairement liés au degré d'information des différents utilisateurs.

Les marchands « internationaux » parviennent à gérer une telle diversité monétaire $^{3}$. Les trésors monétaires et les inventaires après décès témoignent également de la diversité des pièces en circulation à la fin du $\mathrm{XV}^{\mathrm{e}}$ et au début du $\mathrm{XVI}^{\mathrm{e}}$ siècle, mais il n'est pas certain que le phénomène soit maîtrisé par l'ensemble de la population. Ainsi, en $1476^{4}$, un convoyeur de deniers des impositions royales montre imprudemment ce qu'il transporte dans une auberge à Tulle et se fait dépouiller d'une partie lors de changes abusifs. Plusieurs témoins précisent qu'ils ont alors vu des écus (français), des saluts (anciens), des nobles (anglais) et beaucoup d'autres monnaies dont ils ne savent pas «spécifier» les noms (et sans doute encore moins les valeurs) ${ }^{5}$.

A cette date, cependant, des évaluations des monnaies anciennes ou étrangères sont régulièrement annexées aux ordonnances monétaires. Les administrations ont produit des tarifs de plus en plus étoffés au cours du $X^{\mathrm{e}}$ siècle qui ont abouti, aux Pays-Bas à des placards imprimés dès 1487 et accompagnés de gravures dès $1499^{6}$. En France, les quelques pièces anciennes ou étrangères citées dans les ordonnances monétaires du $\mathrm{XIV}^{\mathrm{e}}$ siècle laissent la place à des listes qui s'allongent dans les années 1460 pour compter, à partir de 1473, une quarantaine de pièces d'or et autant de pièces d'argent. Au même moment, des ordonnances comparables apparaissent en Savoie en 1483 ou en Bretagne en $1488^{7}$.

Un examen attentif montre que d'une ordonnance à l'autre, d'un paiement à l'autre, les rapports de valeur entre les différentes pièces ne sont pas tout à fait

\footnotetext{
2 Arch. nat., JJ 270, fol. 406.

3 Archibald (1983); Grierson (1979).

4 Arch. nat., Z1b 480, $\mathrm{n}^{\circ} 4$.
}

5 Un hôtelier parle de «nobles, saluts, reaux de chaz, florins et autres pieces estranges que ne savoit bien specifier », et sa femme parle de « grands nobles et plusieurs pieces que ne saurait specifier car elle ne les connoit pas». En revanche, le receveur, qui en a changé et détourné certaines, décrit une quinzaine de pièces d'or : «saluts, royaux, ecus du coin du roi, de Toulouse, de Guienne, Bretagne, Savoie, de Foix, de Bourbon et autres sortes, florins au chat, du Trait, d'Aragon, pacifics, ducatz, nobles, angelots... » Il dispose de plus d'une pierre de touche qui lui permet de se faire une idée du titre de ces pièces.

6 Il y en eut sans doute plus tôt en Allemagne, puisque, par exemple, le cabinet des médailles de Lausanne conserve une feuille d'ordonnance imprimée à Ulm dans les années 1470 avec des gravures.

7 Références dans Bompaire et Dumas (2000), p. 305. 
constants et que les variations ne sont pas rigoureusement proportionnelles, même en prenant en référence la monnaie la plus stable, le ducat d'or de Venise (ou ses équivalents : florin de Florence et autres ducats) ${ }^{8}$.

Les cours qui y sont indiqués ne reflètent pas exactement le contenu de métal précieux tel qu'on peut le calculer à partir des données des ordonnances de fabrication (étaient-elles respectées ?) ou même des procès-verbaux de fabrication qui mentionnent d'éventuelles faiblesses de titre ou de poids (quelle était la précision de ces mesures ?) ni même à partir des analyses métalliques modernes. Tel est le constat, telle est la question ici envisagée : pourquoi et comment de tels écarts ontils pu s'introduire dans les évaluations?

Certaines décotes et surcotes reflètent des intentions politiques des rédacteurs :

- Il s'agit d'abord de favoriser la monnaie locale officielle qui est, bien sûr, toujours surévaluée.

- On pense aussi à une demande particulière pour des monnaies de bonne réputation, les monnaies qui servent de support au change par lettre, avec un écho des variations qui affectent ce marché..., mais il est bien difficile d'en apporter la démonstration précise, même à partir des données réunies à l'échelle européenne par Spufford (1986) ${ }^{9}$.

- Le fonctionnement des systèmes de compte conduit aussi à des distorsions : on compte, par exemple, 5 florins pour 4 francs en Languedoc après 1360, mais il s'agit d'une équivalence approchée qui favorise plutôt (selon la loi de Gresham) la circulation du florin, bien ancré dans le système de compte régional. Le rapport avec l'ancien gros d'argent (de compte) et avec le ratio légal or/argent expliquent aussi comment ce système un peu déséquilibré a pu fonctionner durant une quinzaine d'années.

- Des équivalences comparables conduisent à donner alors aux anciens écus une valeur sous-évaluée de 2/3 de franc alors que le contenu métallique était au minimum (pour l'émission la plus affaiblie) de $78 \%$ de celui du franc; du coup, l'écu disparaît rapidement des comptes et des trésors. L'écu avait déjà été sous-évalué à la suite de ses affaiblissements successifs et secrets qui l'avaient amené à un titre de 18 carats (75\% d'or). Or, le chroniqueur Jean le Bel évoque avec méfiance ces écus « dont la tierce partie était de cuivre », ce qui laisse entendre qu'il les estimait au titre de $66 \%$ !

- La réputation, justifiée ou non, des pièces apparaît comme un élément décisif : Grierson (1988) ${ }^{10}$ a expliqué comment la présence d'un grand nombre d'imitations de ducats en Egypte avait amené leur décote et conduit des historiens de cette région à considérer le ducat de Venise comme une pièce de bas titre.

${ }^{8}$ En prenant en considération des rapports on échappe aux objections éventuelles liées à l'expression des cours en monnaie de compte liée à la monnaie d'argent et donc aux fluctuations de cellesci comme à celles du ratio or/argent.

9 Spufford (1986).

${ }^{10}$ Grierson (1988). 
La liste serait longue de ces écarts de cours et bien des gloses et des discussions seraient nécessaires pour tenter de les expliquer.

\section{L'ÉLABORATION DES ÉVALUATIONS}

La principale explication tient peut-être au caractère empirique de ces tarifs : même les membres des compagnies italiennes, qui pourtant étaient friandes de ce type d'information qu'elles s'échangeaient dans leur correspondance, ne connaissaient pas les conditions d'émission de toutes les pièces frappées en Europe et moins encore de toutes les anciennes pièces qui y avaient été frappées.

Les princes ne les connaissaient pas davantage et on a des témoignages sur les grandes enquêtes d'évaluation des monnaies étrangères lancées en France à l'époque de Charles V-Charles VI, aux Pays-Bas de façon assez régulière au XV siècle $^{11}$ : en 1366 Charles V demande une enquête ${ }^{12}$; en 1434 et 1440, l'administration de Henri VI demande l'analyse de pièces des Pays-Bas, d'Angleterre ou de Bretagne « afin de la preuve de la valeur d'icelles monnaies les unes contre les autres pour le cours d'icelles $»^{13}$.

Ces enquêtes ne diffèrent pas fondamentalement des enquêtes privées que pouvaient pratiquer des changeurs. J'ai pu étudier le livre de changeur tenu à Montpellier par la famille Malart ${ }^{14}$, une famille de changeurs qui eurent à plusieurs reprises la responsabilité de l'atelier monétaire de Montpellier au XV siècle. On y trouve une liste illustrée de monnaies d'or qui présente un riche panorama des monnaies en circulation au début du $\mathrm{XV}^{\mathrm{e}}$ siècle, monnaies qu'ils avaient pu manier et analyser.

Ces livres de changeurs avaient eu un grand succès auprès des numismates des siècles passés. On s'est aperçu au XIX ${ }^{\mathrm{e}}$ siècle que leurs indications ne correspondaient pas toujours aux données des archives de l'administration monétaire et ils ont été relégués parmi les sources les moins fiables et négligés.

Ce sont précisément ces imperfections qui font leur intérêt.

Elles démontrent que l'accès aux informations monétaires était particulièrement difficile et que les contemporains ne bénéficiaient pas comme les historiens actuels des archives monétaires. Leur comportement économique ne doit pas être interprété (et critiqué) par les historiens en fonction des données dont nous disposons mais de celles dont ils pouvaient disposer. J'y reviens plus particulièrement pour le cas de la France royale.

Elles rappellent aussi que l'opération technique de l'essai est complexe et aléatoire : des erreurs sont possibles sur un échantillon réduit ou, a fortiori, sur une seule pièce. Pour l'argent, l'opération de coupellation est décrite dans La Pratica della mercatura du Florentin Pegolotti vers $1340^{15}$ ou dans des livres de chan-

\footnotetext{
11 Van der Wee et Aerts (1980); Boffa (2000); Spufford (1978).

12 Arch. nat., Z1b 56, fol. 60.

13 Arch. nat., Z1b 966.

14 Bompaire (1992).

15 De Pegolotti (1938), p. 344.
} 
geurs du XV $\mathrm{V}^{\mathrm{e}}$ siècle. Les études en cours de L. Calmels ${ }^{16}$ attirent l'attention sur la marge d'incertitude (surtout pour les monnaies de billon) et sur le bénéfice du doute qui était accordé dans une assez large mesure aux responsables de la fabrication $^{17}$. La coupellation ne faisait pas partie des opérations couramment pratiquées par les changeurs : sur les célèbres tableaux de l'école flamande, on ne voit pas le matériel nécessaire aux essais, tout au plus parfois une fiole de réactif pour l'essai à la touche ; et encore pierre de touche et jeux de toucheaux étalonnés étaient apparemment des objets fort rares : la Chambre des monnaies s'en fait voler et a bien du mal à en reconstituer un jeu aussi complet... Autre indice en ce sens, les nombreux exemples de copies de ces listes d'évaluations de monnaies d'un livre de changeur à l'autre (ce qui peut être aussi une source d'erreurs). Le cas le plus spectaculaire me semble être la présence d'une liste, quasiment identique à la base et datée des années 1290-1300, dans le livre du modeste changeur florentin Lipo di Fede vers 1310, mais encore dans la célèbre Pratica della mercatura de Pegolotti, un des principaux agents de la grande compagnie des Bardi vers 1340 et encore vers 1370 dans les archives du marchand Datini de Prato ${ }^{18}$ ! Ces listes, copiées avec respect, étaient des denrées rares et précieuses bien difficiles à mettre à jour avec ses propres capacités pour un marchand.

Dernière observation sur la démarche empirique de ces listes : la liste de la famille Malart illustre six variétés de francs de Charles V sans trouver de variations de titre significatives. A l'inverse, telle autre liste décrit pour un même type toute une gradation de titres, le rédacteur n'a pas été capable de discerner sur les pièces les marques (secrètes) qui pouvaient indiquer le changement d'émission : « autres semblables, plus durs a la main et ne les connoist on que a veue d'œul... » Il était dans la même position que le numismate moderne (avant l'apparition des analyses non-destructives).

\section{L'INFORMATION \\ SUR LE TITRE DES MONNAIES FRANÇAISES A L'ÉPOQUE DES MUTATIONS}

Pour la France royale, j'ai déjà évoqué l'illusion d'optique à laquelle conduit la consultation des archives monétaires publiées par F. de Saulcy ${ }^{19}$. Il faut bien considérer les informations dont pouvaient disposer d'une part le public en général, d'autre part les changeurs et spécialistes fréquentant les ateliers monétaires et enfin les rares « initiés » qui avaient accès aux actes de l'administration. Cet examen est particulièrement concluant dans la période d'instabilité des grandes mutations monétaires des années 1330-1360 quand le gouvernement des monnaies visait avant tout à procurer des ressources aux finances royales ${ }^{20}$.

\footnotetext{
16 Calmels (2000, 2002).

17 Gandal et Sussman (1997).

18 De La Roncière (1973); Grierson (1979) ; Ciano (1964).

19 de Saulcy (1879-1892).

${ }^{20}$ Cf. en dernier lieu Bompaire (2000).
} 
La publication des ordonnances monétaires royales, le cri des monnaies, se fait solennellement à tous les carrefours et dans tous les bourgs : elle ne mentionne que le nom et le cours des pièces.

Les changeurs et marchands fréquentant l'atelier y sont convoqués pour recevoir une information complémentaire. Ils sont informés :

- du tarif d'achat du marc d'argent,

- du titre du billon qu'ils doivent fournir pour alimenter le fonctionnement de l'atelier.

A partir de ces seules informations, les changeurs concluent que c'est le titre de la fabrication en cours. Ils pèsent les pièces pour connaître leur poids. Ils scrutent les pièces pour découvrir les marques d'émissions et autres «points secrets» qui ne leur sont pas indiqués.

Un marchand-changeur de Montolieu (Aude) vers $1340^{21}$ tombe dans les pièges ainsi tendus :

- il croit que le titre change précisément au moment où le tarif du métal change ;

- il ne sait pas qu'à certains moments le titre réel est abaissé par ajout de cuivre : de janvier à mai 1349, il annonce un titre ${ }^{22}$ de $2 \mathrm{~d} .12$ gr. alors que les pièces sont à 2 d. 5 gr. depuis janvier (17,7\% au lieu de 19,9\% de fin);

- il ne dit rien sur le pied de monnaie ${ }^{23}$ qui n'était pas davantage communiqué à partir de cette date, sauf au moment des renforcements qui marquaient la fin d'un cycle d'affaiblissements ${ }^{24}$;

- il s'efforce de décrire les différents d'émission, mais il s'agit toujours d'ajouts portés sur la liste des cours de l'argent qui, elle, est clairement issue des informations prises à l'atelier monétaire de Toulouse. De plus, selon le classement

21 Bompaire (1987).

${ }^{22}$ Le titre s'exprime pour l'argent en douzièmes ou deniers : chaque denier de loi est subdivisé en 24 grains représentant $1 / 288^{\mathrm{e}}$ de fin, soit un peu moins de 4 millièmes.

${ }^{23}$ Ce chiffre permet de comparer les contenus métalliques des différentes monnaies. Le pied 12 correspond à une fabrication de 3 livres ou 60 sous de monnaie à partir d'un marc d'argent fin. C'est le cas du gros tournois frappé en argent fin au poids de $1 / 60^{\mathrm{e}}$ de marc pour un cours de 12 deniers tournois. En 1341 le gros à la fleur de lis valant 15 d. t. n'était qu'au demi-fin (6 d. de loi) et on en frappait 84 pièces au marc, soit 168 avec un marc d'argent fin qui représentaient une valeur de 168 x $15=2520$ d. t. soit 210 sous ou 10,5 livres : le pied était 42 .

${ }^{24}$ Les exercices de calcul contenus dans les livres de changeurs montrent que l'on savait déduire le titre de pièces quand on connaissait leur pied, leur cours et leur poids.

Par exception, on trouve en janvier 1349, la formule « Ce jour fu dicte la creue aux marchans de 5 solz tournois au blanc pour faire des gros tournois et les doubles de 2 tournois sur le pié de monnoie $36^{\mathrm{e}} »$, mais c'est peut-être un aide-mémoire du greffier plutôt que la retranscription textuelle de cette criée.

Dans la période antérieure, jusqu'en 1343, l'indexation se faisant sur le cours du gros tournois, c'est-à-dire sur le pied de monnaie, celui-ci était peut-être plus souvent publié ; il figure assurément jusqu'en 1346 sur la liste des valeurs du marc d'argent du ms. Arch. nat., KK 953. 
proposé par Duplessy $(1998)^{25}$, il lui arrive de prendre une variante typologique secondaire pour une marque de changement d'émission... ${ }^{26}$

A certains moments, le roi (ou plutôt le souverain maître des monnaies Jean Poillevillain) autorise les ateliers à acheter du cuivre et, en même temps, de l'argent fin à ses frais. Il s'agit de ne pas retarder l'ouvrage si des marchands apportent du métal d'un titre trop élevé ou d'un titre trop bas. Je me suis laissé prendre à interpréter en terme de conjoncture ces mesures contradictoires : y avait-il trop de bas billon ou de billon de haute loi ? L'explication est ailleurs. A partir de ce moment les changeurs les plus méfiants ne pouvaient plus déterminer si l'achat de cuivre était lié à un affaiblissement secret.

Les marques sont parfois changées avant les changements de titre ${ }^{27}$. Dans d'autres cas, le tarif d'achat du métal est augmenté avant les affaiblissements de titre. Ceux-ci sont alors accompagnés des nouveaux différents d'émission (l'opération n'aurait plus d'intérêt si les différents étaient immédiatement signalés aux changeurs). Les changeurs qui pratiquaient alors des essais immédiatement ne trouveraient pas de changement de titre lié aux différents dans le premier cas ni à la crue dans le second.

Quand bien même ils poseraient des questions, la Chambre des monnaies conseille aux gardes de mentir effrontément et de dire (en décembre 1359) que des pièces à $3 \mathrm{~d}$. sont à $4 \mathrm{~d}$. : " se aucuns s'apercoit qu'ils sont a $3 \mathrm{~d}$., feignez et maintenez qu'il sont a 4 d. ». C'est le signe que les changeurs douteront plutôt de leur essai privé que de l'atelier monétaire dont la parole doit suffire pour couper court à une rumeur. On a déjà vu à Limoux que de même la parole d'un changeur ou d'un courtier avait une autorité suffisante. De plus, il était difficile aux changeurs d'apporter des éléments à la discussion dans la mesure où il était rigoureusement interdit de fondre la monnaie du roi.

Enfin, à de rares et brefs moments (en novembre 1353 ou 1355 pour l'argent, un peu plus longtemps pour les écus d'or), le secret absolu est imposé sur les émissions et des émissions affaiblies sont faites sans marques distinctives.

Les changeurs ordinaires, les comptables et les thésauriseurs sont piégés. Les prix des monnaies sont fixés par les changeurs et les ordonnances royales n'y peuvent rien, disaient ses conseillers à Philippe le $\mathrm{Bel}^{28}$. En l'occurrence, les changeurs ont pu être dépassés. La valeur à laquelle ces pièces ont été acceptées n'est pas leur valeur métallique véritable qui échappe à la plupart des utilisateurs.

25 Duplessy (1998).

${ }^{26}$ Des discussions devaient prendre corps autour des différents et le 28 juin 1360, lors d'un affaiblissement de titre et de poids, la Chambre des monnaies suggère aux officiers des ateliers monétaires de ne parler que du poids et de dire que «le différent est mis pour cause de la taille afin que les monnayeurs ne puissent mêler les forts avec les faibles », alors que l'usage est de ne pas mettre de différent quand seul le poids est modifié (et cet usage est encore rappelé aux gardes le 6 août suivant).

${ }^{27}$ Par exemple pour les coins des écus d'or de l'émission à 18 carats en 1351 qui servent d'abord à frapper le métal disponible qui avait été préparé à 20 carats.

${ }^{28}$ Avis au roi de Pierre Chief-de-Roy de Limoges en 1313 : « ...car pour le pris que le changeur y donra que la monnaie que ce soit, ele se metra et si la met au-dessouz, au dessous ira, et se la met au dessus, au dessus ira et se mettra pour le pris que le changeur i metra » : Guilhiermoz (1925), p. 97. 


\section{DES INDEXATIONS PARTIELLES ET IMPARFAITES}

Et pourtant les contemporains pratiquaient régulièrement une indexation des prix sur le métal précieux dont on a maints exemples. On a observé depuis longtemps que cette pratique aurait dû réduire à néant l'effet et le profit des mutations, au moins vis-à-vis des changeurs. On pouvait au moins supposer, en effet, que ceux-ci profitaient de l'ignorance du grand public pour diffuser la monnaie faible au prix fort, comme notre drapier catalan quittant Toulouse pour Limoux. On avait néanmoins du mal à concevoir comment le système des mutations pouvait perdurer et se reproduire avec succès (et profit) une fois que les bourgs, les villages et les campagnes étaient saturés de mauvaises monnaies : qui payait ? demandait avec raison Alain Guerreau ${ }^{29}$.

En fait, même les changeurs ne disposaient que d'un élément solide sur la valeur du métal, le tarif d'achat du marc d'argent et c'est celui-ci qui servait d'ailleurs officiellement de base pour les évaluations de dettes anciennes à la Chambre des comptes ou au Parlement. Les prix ne suivent pas tant l'évolution du contenu réel des monnaies (bien mal connu) que celle du tarif d'achat officiel du métal.

Le manuscrit de la famille Malart de Montpellier me permet d'illustrer ce propos : il contient les cours mensuels de deux monnaies d'or (l'écu et le mouton) à Montpellier, cours que l'on peut comparer à l'évolution du tarif d'achat du marc d'argent et à celle du rendement du marc d'argent à l'émission, de la traite, au moins pour les années 1417-1422, pour lesquelles les comptes de l'atelier sont conservés.

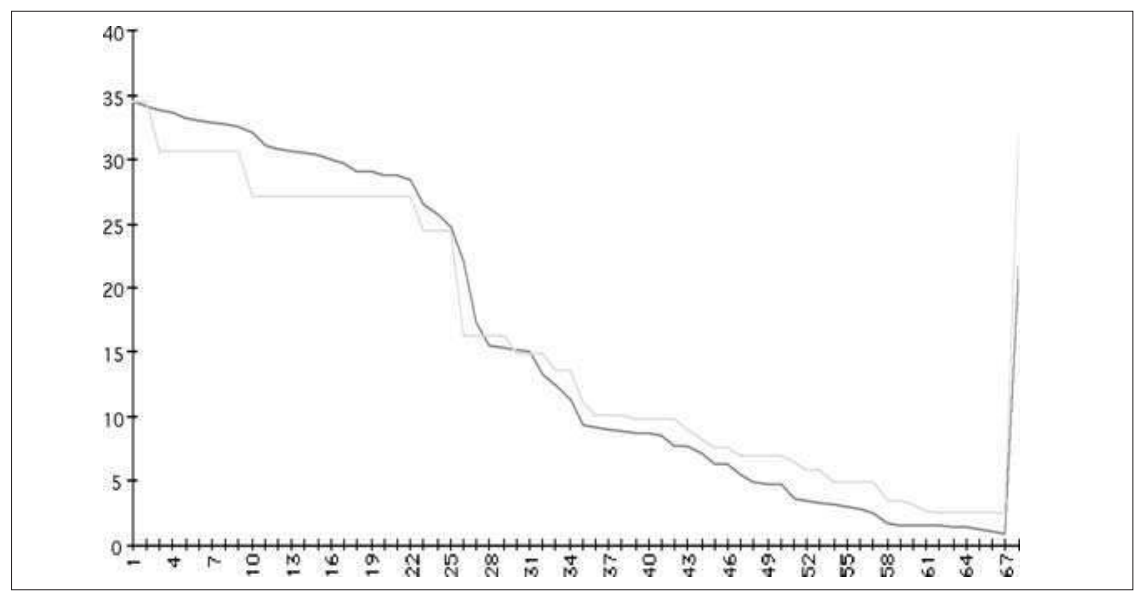

Figure 1. Evolution comparée du contenu d'argent fin (en grammes) représenté par une livre tournois, calculé à partir du tarif d'achat du métal à l'atelier monétaire de Montpellier, et du contenu d'or fin (en décigrammes) représenté par une livre tournois calculé à partir du cours du mouton d'or relevé à Montpellier.

${ }^{29}$ Guerreau (1987). 


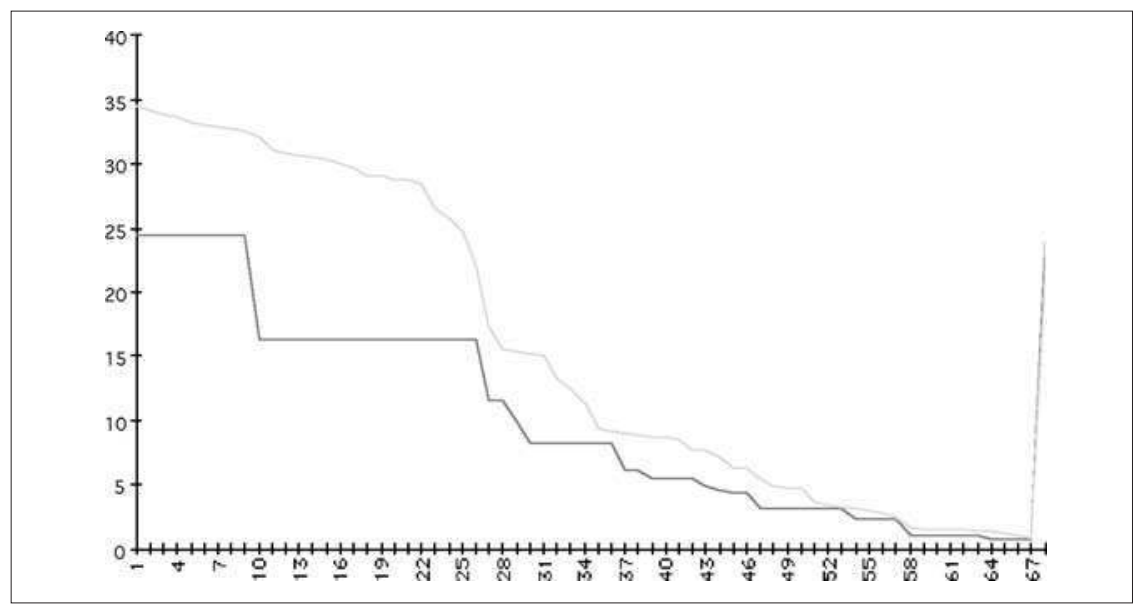

Figure 2. Même comparaison calculée à partir de la traite, c'est-à-dire du rendement à l'émission, de la valeur fabriquée à partir d'un marc d'argent.

N. B. Ces deux figures font l'hypothèse d'un ratio or/argent de 10.

L'écart entre les deux valeurs, tarif et traite, correspond aux frais de fabrication et surtout au prélèvement du seigneuriage royal qui pouvait s'élever jusqu'à $40 \%$ de la valeur de la pièce dans cet épisode de «grande inflation». Les changeurs y avaient souvent leur part sous la forme « d'avantages », c'est-à-dire de prix d'achat du métal supérieurs au tarif officiel, qui leur étaient parfois consentis.

La priorité accordée par les contemporains au tarif par rapport à la traite (qui restait inconnue en période de mutations) est d'ailleurs prouvée par l'évolution de la production dans les ateliers monétaires : le métal afflue lorsque le tarif d'achat est augmenté, indépendamment de l'évolution de la traite ou du taux de prélèvement. Elle s'explique assez bien par le manque d'informations disponibles et par l'incapacité de la plupart des changeurs à les vérifier et à les compléter par eux-mêmes.

Les mutations monétaires permettaient ainsi de dégager une marge de bénéfice pour le roi et pour les responsables des ateliers monétaires et pouvaient donc se poursuivre.

Plutôt que des experts, il y a donc des initiés : les maîtres des ateliers monétaires, souvent membres de familles, de compagnies, de réseaux de changeurs qui bénéficient ainsi d'informations privilégiées. C'est par leur intermédiaire, probablement, que les informations se diffusaient.

Il faut y ajouter les personnes présentes au conseil du roi où les décisions sont prises. Ainsi, en 1342, les imitations des monnaies royales frappées par le comte de Savoie sont un bon reflet des émissions royales (dont les conditions sont rigoureusement reprises) parce que le comte était présent au conseil avec le roi : quand il veut, parallèlement, connaître les conditions d'émission des monnaies de Milan, pour les imiter de même, il ne fait pas essayer les monnaies milanaises, mais il 
envoie des émissaires s'informer dans cet atelier monétaire ${ }^{30}$. C'est apparemment à ses yeux la seule façon de recueillir une information fiable. Le duc de Bourgogne envoie de même un émissaire pour savoir à quel titre frappe l'atelier du roi à Troyes en $1359^{31}$.

Les processus d'imitation des monnaies d'un prince par un autre me paraissent devoir également être reconsidérés à la lumière de cette sous-information. Il ne s'agit pas nécessairement de contrefaçons frauduleuses, de recherche du gain ou de guerres monétaires. Certes, j'ai pu observer une course à l'affaiblissement du titre des florins entre la Provence et la principauté d'Orange vers $1360^{32}$. J'aurais peut-être dû souligner que les deux princes étaient alors liés, le prince d'Orange étant sénéchal de Provence et que les responsables des deux ateliers monétaires étaient probablement aussi liés puisque tous deux florentins. Tout ne doit peut-être pas s'interpréter en terme de guerre monétaire et de loi de Gresham.

\section{BIBLIOGRAPHIE}

Archibald, Marion, «Coinage in Andrew Halyburtton's Ledger», pp. 263-301, in : Studies in numismatic method presented to Philip Grierson, Cambridge, 1983.

Barrandon, Jean-Noël, BompaIRE, Marc, « Les imitations de florins dans la vallée du Rhône au XIV siècle », Bibliothèque de l'Ecole des Chartes, 150, 1989, pp. 141-200.

Boffa, Serge, « Liste provisoire des sources éditées de l'histoire monétaire brabançonne jusqu'en $1430 »$, Revue Belge de Numismatique, 145, 2000, pp. 31-137.

Bompaire, Marc, «Un livre de changeur languedocien du milieu du XIV X $^{\mathrm{e}}$ siècle », Revue numismatique, 1987, pp. 118-183.

Bompaire, Marc, «Les monnayages d'or du $\mathrm{XV}^{\mathrm{e}}$ siècle : le témoignage d'un livre de changeur languedocien », Bulletin de la Société nationale des antiquaires de France, 1992, pp. 354-373.

Bompaire, Marc, «Le gouvernement des monnaies de Jean Poillevillain », in : Guerre, pouvoir et noblesse au Moyen Âge, Mélanges en l' honneur de Philippe Contamine, Paris, 2000, pp. 101-110.

Bompaire, Marc, Dumas, Françoise, Numismatique médiévale, monnaies et documents d'origine française, (L'atelier du médiéviste, 7), Turnhout, Brepols, 2000.

Bompaire, Marc, Geneviève, Vincent, « Une imitation du gros à la fleur de lis de Philippe VI au nom d'Aimon, comte de Savoie, trouvée à Toulouse », Bulletin de la société française de numismatique, 55, 2000, pp. 50-53.

Calmels, Laurence, « Problèmes posés par les essais d'argent à la fin du Moyen Âge », Bulletin de la société française de numismatique, 55, 2000, pp. 120-124.

Calmels, Laurence, « La précision des essais d'argent remise en question : les papiers d'Arcet à la Monnaie de Paris », Revue Numismatique, 2002.

Ciano, Cesare, La «practica della mercatura» datiniana (secolo XIV), (Biblioteca della Rivista Economia e storia, IX), Milan, A. Giuffré, 1964, XII-255 p., pp. 86-91.

Dumas-Dubourg, Françoise, Le monnayage des ducs de Bourgogne, Louvain-la-Neuve, 1984.

Duplessy, Jean, Les monnaies royales françaises de Hugues Capet à Louis XII, t. I, Paris, 1998, $\mathrm{n}^{\circ} 272 \mathrm{~A}$

Gandal, Neil, Sussman, Nathan, « Asymetric information and commodity money : tickling the tolerance in Medieval France», Journal of Money, Credit and Banking, 29, $\mathrm{N}^{\circ}$ 4, 1997, pp. 440-457.

\footnotetext{
30 Bompaire et Geneviève (2000).

31 Dumas-Dubourg (1984).

32 Barrandon et Bompaire (1989).
} 
Grierson, Philip, «Coinage in the Cely papers», in : Later Medieval Numismatics, Variorum, Londres, 1979, XV, repris de Miscellanea medievalia F. Niermeyer, Groningue, 1967, pp. 375-404.

Grierson, Philip, «The Coin List of Pegolotti», in : Later Medieval Numismatics, Variorum, Londres, 1979, XI, repris de Studi in onore di Armando Sapori, Milan, 1957, vol. 1, pp. 485-492.

Grierson, Philip, «The fineness of the Venetian ducat and its imitations », pp. 95-104, in : Metallurgy in Numismatics, vol. 2, Londres, 1988.

Guérard, Léon, Documents pontificaux sur la Gascogne tirés des archives du Vatican, t. II, Paris, 1903 (Archives historiques de Gascogne, $2^{\mathrm{e}}$ série, fasc. 6).

Guerreau, Alain, «Quelques réflexions sur les mutations monétaires en France à la fin du Moyen Âge ", in : Rythmes de la production monétaire de l'Antiquité à nos jours, Louvain-la-Neuve, 1987, pp. 521-535.

Guilhiermoz, Paul, « Avis sur la question monétaire donnés aux rois...», Revue numismatique, 1925.

La Roncière (de), Charles, Un changeur florentin du trecento, Lippo di Fede del Sega, 1285 environ1363 environ, Paris, SEVPEN, 1973, 278-2 p. (Affaires et gens d'affaires, 36).

Pegolotti (de), Francesco Balduccio, La practica della mercatura, Evans, Allan, ed., Cambridge, Mass., 1938, § 87.

Saulcy (de), Félicien, Recueil de documents inédits relatifs à l' histoire des monnaies frappées par les rois de France, 4 vol., Paris, 1879-1892.

Spufford, Peter, Monetary problems and policies in the Burgundian Netherlands (1433-1496), Leyde, 1978, pp. 117-127.

Spufford, Peter, Handbook of medieval exchange, Londres, 1986, 376 p.

Van der Wee, Hermann, Aerts, Erik, « Het geholde van de Coudmunten in Vlaanderen en Brabant tijdens de late Mideleeuwen », Revue Belge de numismatique, 125, 1980, pp. 129-157. 